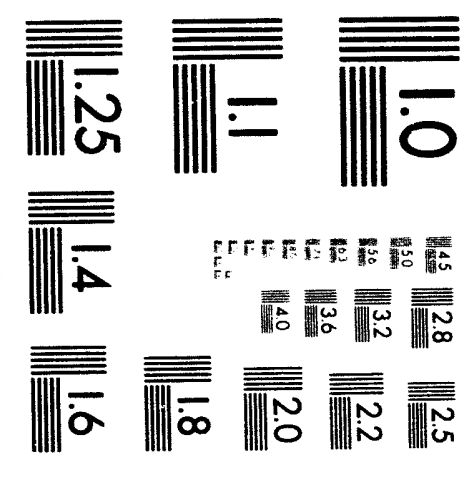






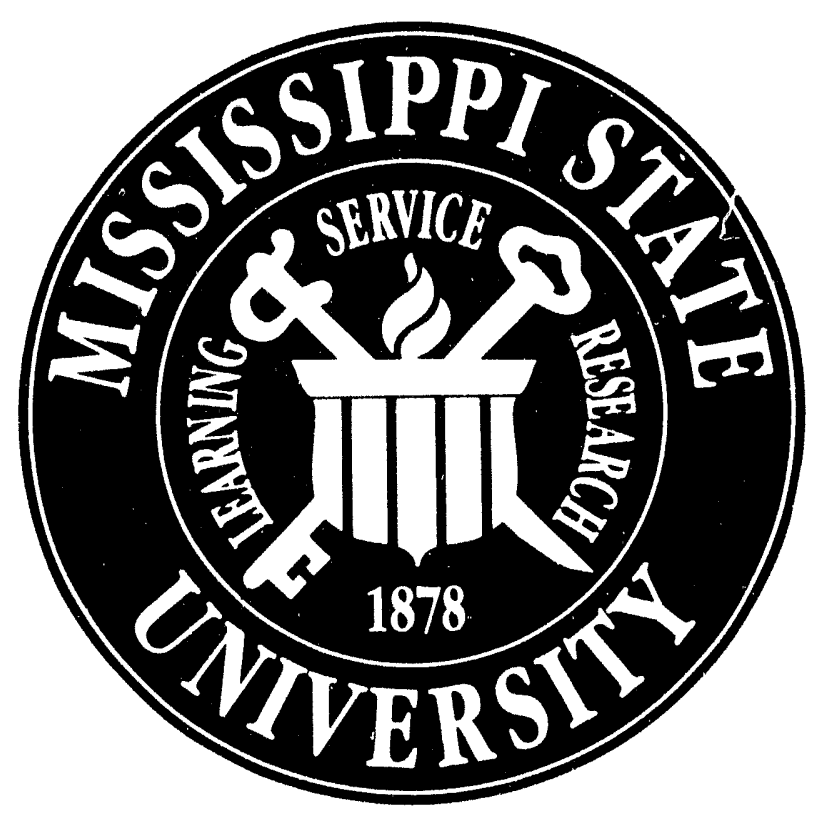

\section{DIAGNOSTIC INSTRUMENTATION AND ANALYSIS LABORATORY}

COHERENT ANTI-STOKES RAMAN SPECTROSCOPY SYSTEM FOR POINT TEMPERATURE AND MAJOR SPECIES CONCENTRATION MEASUREMENTS

\section{Topical Report}

Jagdish P. Singh and Fang-Yu Yueh

Prepared for the United States

Department of Energy

Under Contract No. DE-AC02-80ET-15601 
FE-15601

Topical Report 11

\title{
COHERENT ANTI-STOKES RAMAN SPECTROSCOPY SYSTEM FOR POINT TEMPERATURE AND \\ MAJOR SPECIES CONCENTRATION MEASUREMENT
}

\author{
Topical Report \\ Jagdish P. Singh and Fang-Yu Yueh \\ Diagnostic Instrumentation and Analysis Laboratory \\ Mississippi State University
}

Prepared For The

U.S. Department of Energy

Contract No. DE AC()2-80ET-15601

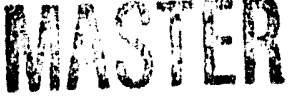


This report was prepared as an account of work sponsored by the United States Government. Neither the United States nor the United States Department of Energy, nor any of their employees, makes any warranty, expressed or implied, or assumes any legal liability or responsibility for the accuracy, completeness, or usefulness of any information, apparatus, product, or process disclosed, or represents that its use would not infringe privately owned rights. Reference herein to any specific commercial product, process, or service by trade name, mark, manufacturer, or otherwise, does not necessarily consittute or imply its endorsement, recommendation, or favoring by the United States Government or any agency thereof. The views and opinions of authors expressed herein do not necessarily state or reflect those of the United States Government or any agency thereof. 
TABLE OF CONTENTS

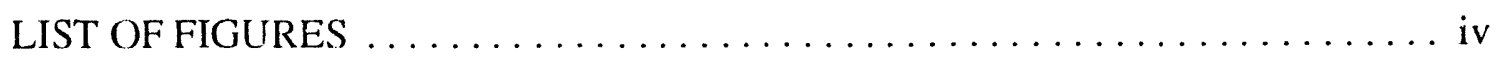

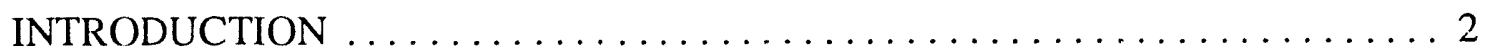

PURPOSE OF THE INSTRUMENT $\ldots \ldots \ldots \ldots \ldots \ldots \ldots \ldots \ldots \ldots \ldots \ldots$

PERFORMANCE REQUIREMENTS $\ldots \ldots \ldots \ldots \ldots \ldots \ldots \ldots \ldots \ldots \ldots$

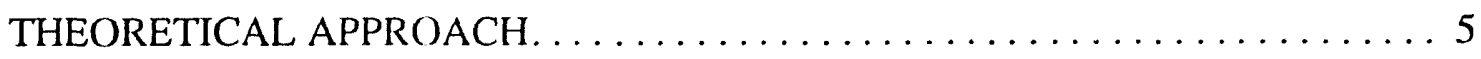

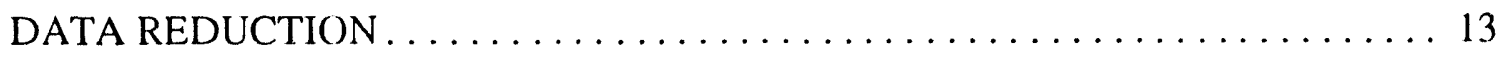

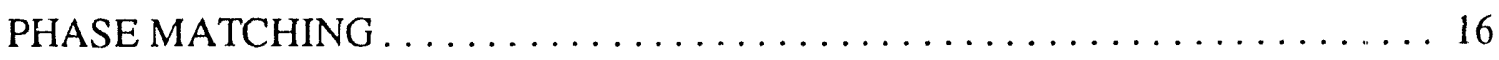

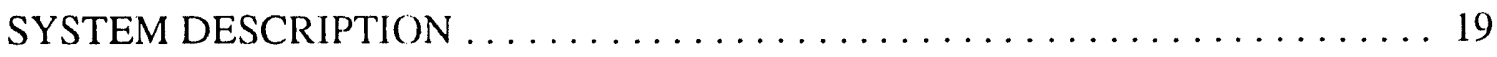

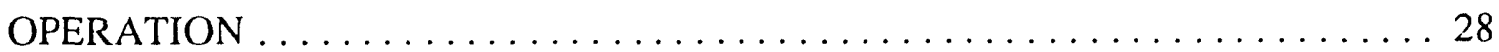

TEMPERATURE AND CONCENTRATION MEASUREMENT $\ldots \ldots \ldots \ldots \ldots 30$

ACKNOWLEDGEMENTS . . . . . . . . . . . . . . . . . . . . . . . . 39

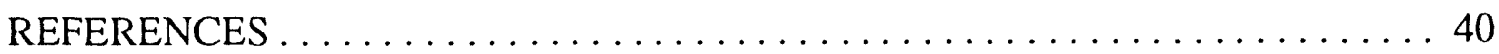




\section{LIST OF FIGURES}

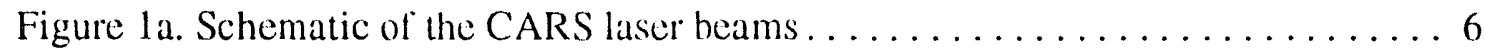

Figure $1 b$. Energy Level Diagram showing CARS process . . . . . . . . . 6

Figure 2. Simulated N2 CARS spectra at different temperatures.......... 7

Figure 3. Simulated CO CARS spectra at different concentrations . . . . . . . 8

Figure 4. CARS phase matching geometries $\ldots \ldots \ldots \ldots \ldots \ldots \ldots \ldots$

Figure 5. Configuration for the Mobile CARS Instrument. . . . . . . . . 20

Figure 6 . A block diagram of the CARS data acquisition/analysis system $\ldots \ldots \ldots 21$

Figure 7. Arrangement of the Mobile CARS system components . . . . . . . 26

Figure 8. Folded Boxcars spectrum from the CFFF diffuser during May/June 1990 test run

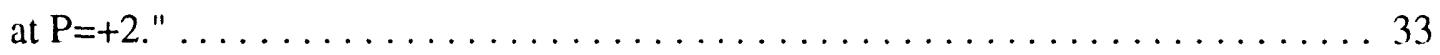

Figure 9. Temperature profile of the CFFF diffuser during the LMF4-T test. . . . . . 34

Figure 10. CARS spectrum recorded at the aerodynamic duct during the LMF5-G test run.

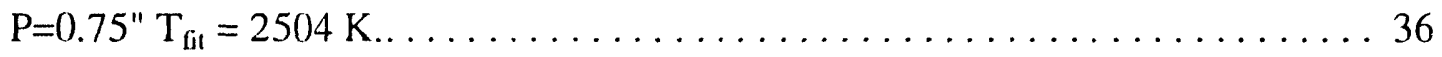




\section{COHERENT ANTI-STOKES RAMAN SPECTROSCOPY SYSTEM \\ FOR POINT TEMPERATURE AND \\ MAJOR SPECIES CONCENTRATION MEASUREMENT}

Topical Report

Jagdish P. Singh and Fang-Yu Yueh

Abstract

The Coherent anti-Stokes Raman Spectroscopy system (CARS) has been developed as a laser-based, advanced, combustion-diagnostic technique to measure temperature and major species concentration. The principles of operation, a description of the system and its capabilities, and the operational details of this instrument are presented in this report. 


\section{INTRODUC'TION}

Coherent Anti-Stokes Raman Spectroscopy (CARS) as a laser-based, advanced, diagnostic technique has drawn the attention of the scientific community because of its potential for practical application. ${ }^{1,2}$ It provides nonintrusive, temporal, and spatial measurement of temperature and major species concentrations in a hostile, turbulent, and harsh combustion environment. The CARS theory and its applications to various combustions can be found in several articles. ${ }^{1-3}$ In brief, CARS is a four wave mixing process in which two pump photons of frequency $\omega_{p}$, and one Stokes photon of frequency $\omega_{s}$ interact through the third order nonlinear susceptibility of a medium and produce a fourth photon at the anti-Stokes frequency of $\omega_{\mathrm{as}}=2 \omega_{\mathrm{p}}-\omega_{\mathrm{s}}$. CARS application has been demonstrated in an internal combustion engine, ${ }^{4}$ a coal gasifier, ${ }^{5}$ a coal-fired MHD simulator, ${ }^{6}$ a coal furnace, ${ }^{7}$ a coal seeded burner, ${ }^{8}$ and an augmented jet engine exhaust. ${ }^{9}$ Most of these measurements have been performed with a CARS instrument in a laboratory or relatively vibration-free environment. In some cases, CARS systems have been tested by keeping the transmitter close to combustors from which personnel are restricted during operation for safety reasons. ${ }^{910}$ In such a situation, any change or repair in the CARS instrument is impossible while the test facility is in operation.

In some practical combustion environments, it is not always possible to keep the CARS system close to the test facility because of limited space and greater vibrations near the facility. A mobile CARS system has been developed for application at this type of facility. The instrument is versatile and with slight modifications can be used with any combustor. This topical report describes the theory and application of the mobile CARS system for measuring the temperature profile and the major species concentrations of a laboratory burner, the DIAL/MSU test stand, and the CFFF facility. The report also addresses the various techniques tested in those measurements, the problems encountered in the tests, and the problems' solutions. 


\section{PURPOSE OF THE INSTRUMENT}

CARS is a well-established, laser-based, combustion-diagnostic technique used to measure temperature and major species concentration in a harsh, turbulent, highly luminous combustion environment. In the past, the technique has usually been limited to the laboratory combustion environment. Few attempts have been made to apply CARS to a large-scale combustion facility.

The CARS instrument developed at the Diagnostic Instrumentation and Analysis Laboratory (DIAL) at Mississippi State University (MSU) is a versatile, mobile CARS system with unique features that suit it for use in a coal-fired, large-scale, magnetohydrodynamic (MHD) combustion facility. Among these features is the capability to make measurements in the presence of coal particles in the gas stream. The instrument has been used to measure temperatures from $300 \mathrm{~K}$ to $2700 \mathrm{~K}$, but mobile CARS can theoretically measure higher temperatures. The concentration measurement range ranges from $1-30 \%$ depending on the species and combustion environment. Accuracy of the temperature measurement is $\sim 1 \%$ in a clean flame and $\sim 5 \%$ in a coal particle loaded flame. 


\section{PERFORMANCE REQUIREMENTS}

The following are the general requirements to apply the mobile CARS instrument to a combustion facility:

Test Facility

1. Two optical ports across the gas stream should be available to pass the laser beam.

2. Transmission of the CARS laser beam through the ports should be higher than $30 \%$.

3. There should be enough space near the optical ports to mount a $12^{\prime \prime} \times 18^{\prime \prime}$ receiver optics plate and lens traverse system.

4. The mobile CARS instrument trailer should be parked within 100 feet of the test facility.

Temperature Measurement

A major species (with concentrations higher than $30 \%$ ) should be used as a test molecule for CARS temperature measurement; its CARS spectra should be evaluated in a laboratory combustion environment.

Concentration Measurement

Major species concentration measurement of a desired species should be tested in the laboratory. 


\section{THEORETICAL APPROACH}

In the CARS process, two narrow-band laser beams at pump frequency $\omega_{l}$, derived from a pump laser (usually a pulse laser), and one broadband dye laser beam at Stokes frequency $\omega_{2}$ are phase-matched and focused in the gas stream to generate a CARS spectrum at the anti-Stokes frequency $\omega_{3}=2 \omega_{1}-\omega_{2}$ as shown in Figure 1. The coherent, laser-like character of the CARS signal allows all of the generated signal to be collected by proper detector placement. When the frequency difference $\left(\omega_{1}-\omega_{2}\right)$ is close to the frequency of a Raman resonance $\omega_{v}$ of a species in the bulk medium. The third order susceptibility, $\chi_{\mathrm{CARS}}$, of the medium is enhanced. Since the intensity of the anti-Stokes beams depends on the squared modulus of the $\chi_{\text {CARS }}$, a strong CARS signal is produced. $\chi_{\text {CARS }}$ is temperature and concentration dependent. Therefore, the shape of the CARS spectrum of a species can be sensitive to both temperature and species concentration. Figures 2 and 3 demonstrate a calculated temperature dependence of $\mathrm{N}_{2}$ CARS spectra and a calculated CO concentration dependence of CO CARS spectra. By fitting the spectrum shape of an experimental CARS spectrum to the calculated CARS spectrum, the temperature and species concentrations can be obtained. This approach has been the most reliable way to extract accurate temperature and concentration information.

To a large extent, the accuracy of the inferred values of temperature and concentration is determined by the synthetic CARS spectra used to analyze the data. The model employed in our CARS work follows the theory of Yuratich" as modified by Kataoka et al..$^{12}$ and Teets ${ }^{13}$ to account for the effects of finite linewidths of the laser sources, namely 


\section{Approach}

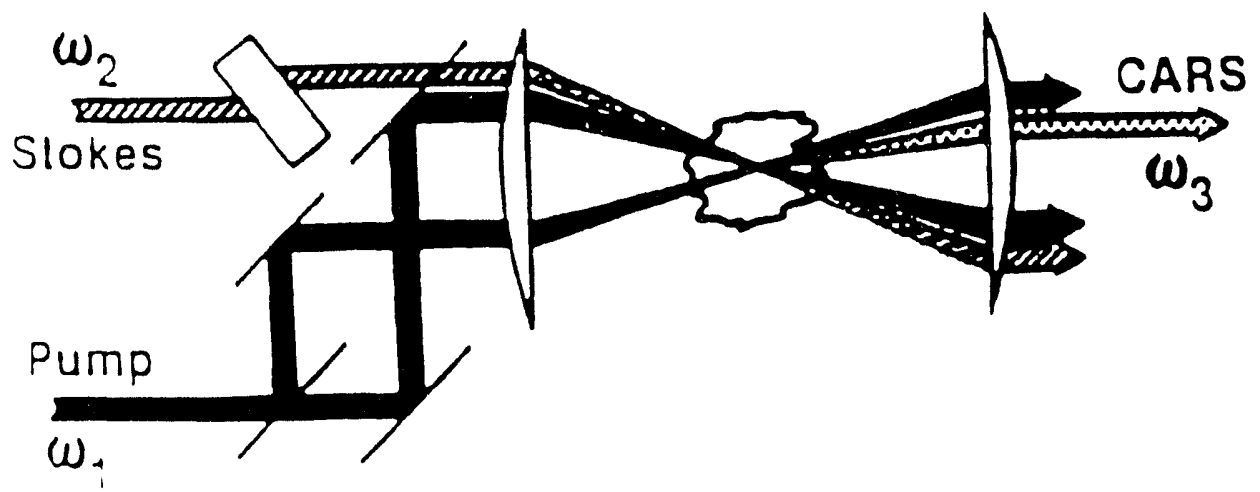

Figure la. Schematic of the CARS laser beams

Energy level diagram

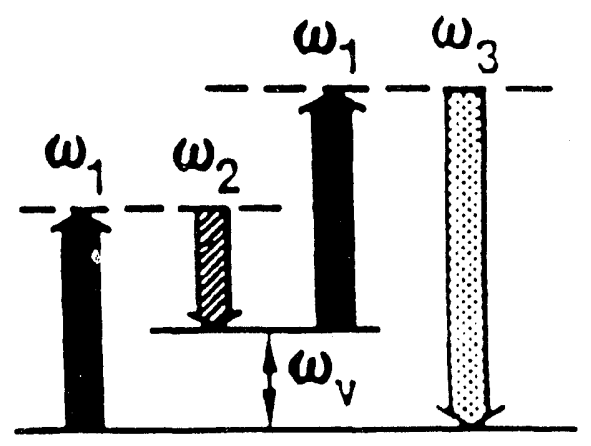

Figure Ib. Energy Level Diagram showing CARS process 


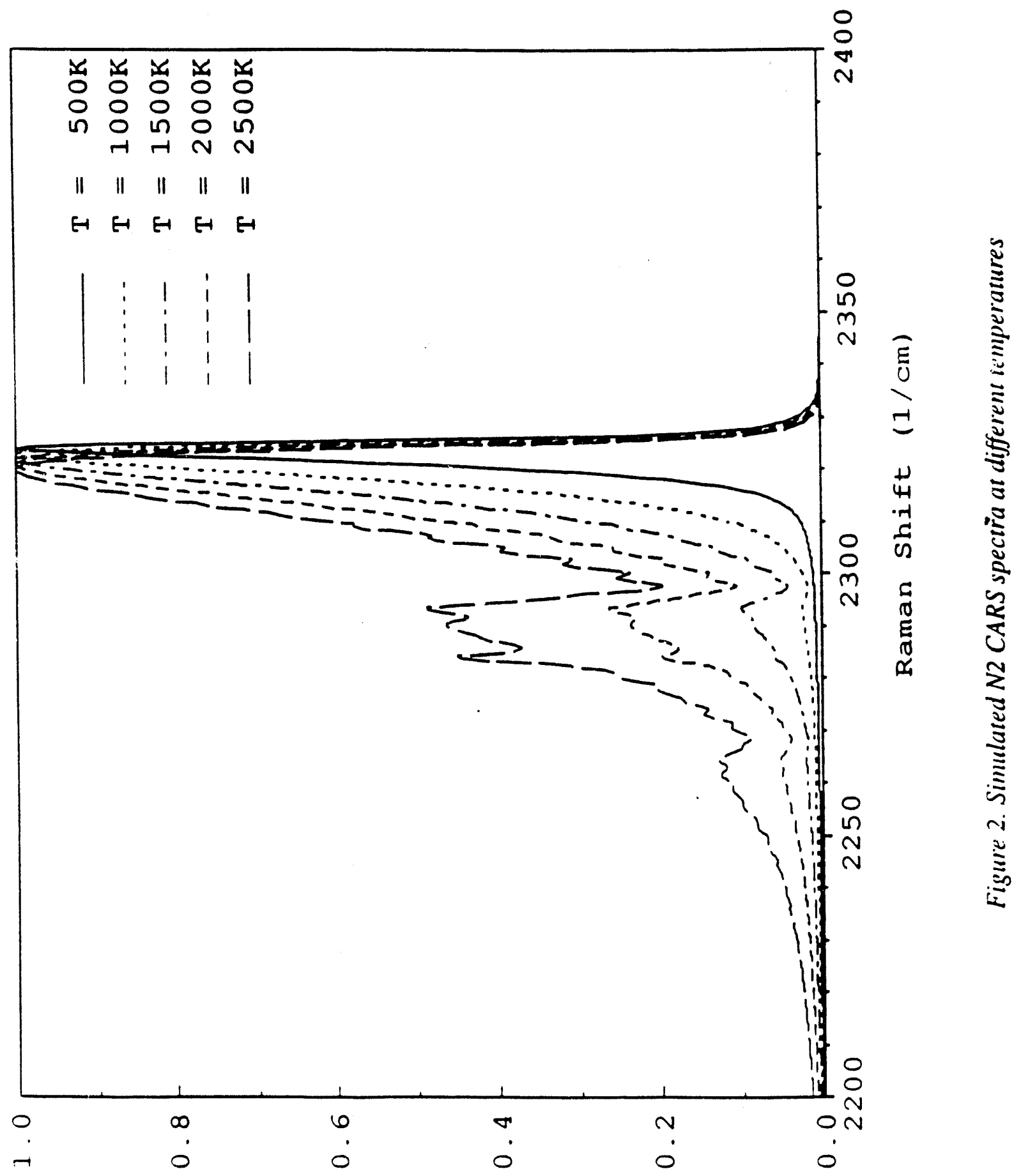




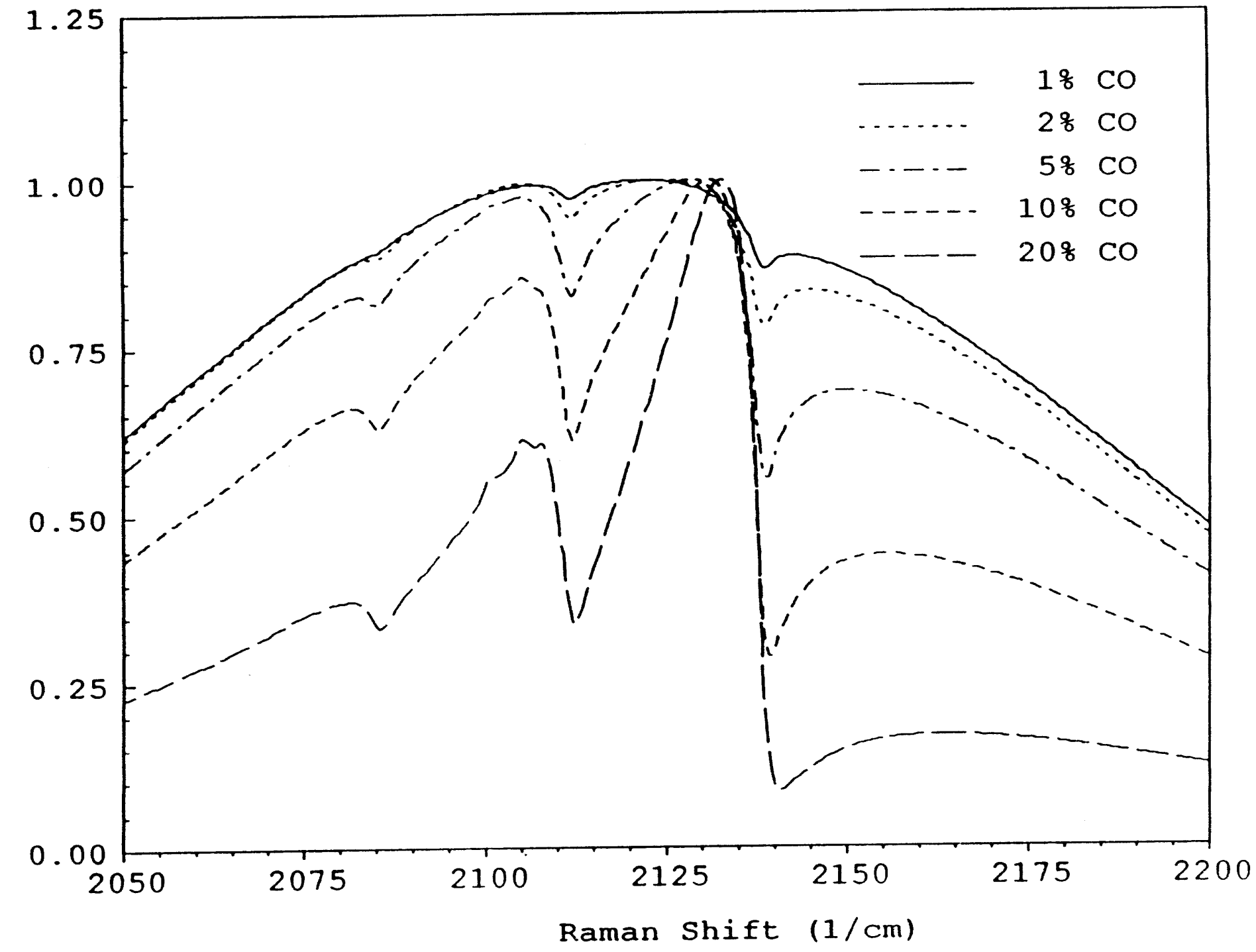

Figure 3. Simulated CO CARS spectra at different concentrations 


$$
\begin{gathered}
\mathrm{I}_{4}\left(\omega_{4}\right)=\frac{16 \pi^{4} \omega_{4}^{2}}{c^{4} n_{1} n_{2} n_{3} n_{4}}\left[\frac{\sin \Delta k z / 2}{\Delta k z / 2}\right]^{2} z^{2} \\
\times \int\left|1 / 2 \chi\left(\omega_{1}-\omega_{2}\right)+1 / 2 \chi\left(\omega_{3}-\omega_{2}\right)\right|^{2} I_{1}\left(\omega_{1}\right) I_{2}\left(\omega_{2}\right) I_{3}\left(\omega_{3}\right) \\
\times \delta\left(\omega_{1}+\omega_{3}-\omega_{2}-\omega_{4}\right) d \omega_{1} d \omega_{2} d \omega_{3}
\end{gathered}
$$

where $I_{i}\left(\omega_{i}\right)$ is the spectral density of laser beam i at frequency $\omega_{i}$ with the index of refraction $n_{i}$. The phase mismatch for wave vectors $k_{i}$ is given by $\Delta k=\left|k_{1}+k_{3}-k_{2}-k_{4}\right|$ for beams interacting at distance $\mathrm{z}$. For phase-matched conditions, $\Delta \mathrm{k}=0$, and the factor in the bracket $=1$. The third order susceptibility is represented by $\chi$. The pump radiation is denoted by $I_{1}$ and $I_{3}$, the Stokes radiation by $I_{2}$, and the anti-Stokes radiation by $I_{4}$. For linear polarization of pump and Stokes beams $\chi\left(\omega_{1}-\omega_{2}\right)$ is given by

$$
\chi\left(\omega_{1}-\omega_{2}\right)=3 \chi_{1111}^{(3)}=3 \chi_{1111}^{n r}+\frac{N_{s} 2 \pi}{h} \sum_{i, f}\left[\Omega_{i, f}-\left(\omega_{1}-\omega_{2}\right)-i \gamma_{i, f}\right]^{-1} \Delta \rho_{i, f} \alpha_{i, f}^{2}
$$

where

$$
\Delta \rho \equiv f_{i}-\left(\frac{2 J_{i}+1}{2 J_{f}+1}\right) f_{f}
$$

is the population factor, $\chi_{1111}^{(3)}$ is the $[1111]$ component of the third order susceptibility with nonresonant part $\chi_{1111}^{\mathrm{nr}}, \mathrm{N}_{s}$ is the number density of the probed molecule for which the summation is taken over all initial and final rovibrational levels with Raman resonances $\Omega_{\text {if }}$ and halfwidths $\gamma_{\text {if }}$. The fractional populations of the initial and final levels are given by $f_{i}$ and $f_{f}$, respectively; $\alpha_{f}$ is the polarizability for a transition from $i$ to $f$. When the spectral 
profiles of the pump and Stokes radiations are Gaussian, the threefold convolution of Equation 1 cannot be evaluated to yield a closed form for multiplex CARS. However, an accurate approximation can be written for this expression requiring only that the pump linewidth be much smaller than the Stokes spectral width. ${ }^{14,15}$ As noted below, this allows the Stokes spectral profile to be separated from molecule-dependent resonant summations. Generalizing expression 4 in Table 1 of reference 15 to the case of multiple species, the convolution in Equation 1 can be expressed:

$$
\int=\left(\chi^{\mathrm{nr}}\right)^{2} \mathrm{~F}\left(\omega_{4}\right)\left[1-\left.\frac{2 \sqrt{\pi}}{\tilde{\gamma}_{1}}\left|m S_{1}\left(\omega_{4}\right)+\frac{\pi}{2 \tilde{\gamma}_{1}^{2}}\right| S_{1}\left(\omega_{4}\right)\right|^{2}-\frac{\sqrt{\pi}}{2 \tilde{\gamma}_{1}} \mathcal{S}_{2}\left(\omega_{4}\right)\right]
$$

where

$$
\begin{aligned}
& S_{1}\left(\omega_{4}\right)=\sum_{s} C_{s} \sum_{j} a_{s j} w\left(z_{s j}\right) \\
& S_{2}\left(\omega_{4}\right)=\sum_{s} \sum_{t} C_{s} C_{t} \sum_{j} \sum_{k} a_{s j} a_{t k}^{*} \times \operatorname{lm}\left[\frac{\left[w\left(z_{s j}\right)+w^{*}\left(z_{t k}\right)\right]}{\left(\Omega_{t k}-\Omega_{s j}\right)+i\left(\gamma_{s j}+\gamma_{t k}\right)}\right] \\
& C_{s}=\frac{f_{s}}{\chi^{n r}} ; f_{s} \equiv \text { mole fraction of species } s \\
& a_{s j}=\frac{N 2 \pi}{h} \Delta \rho_{s j} \alpha_{s j}^{2} \\
& N \equiv \text { composite number density } \\
& z_{s j}=\left(\omega_{4}-\omega_{1}-\Omega_{s j}+i \gamma_{s j}\right) / \tilde{\gamma}_{1} \\
& w\left(z_{s j}\right) \equiv \text { complex error function }
\end{aligned}
$$




$$
\Omega_{s j}=j^{\text {th }} \text { Raman resonant frequency of species s, }
$$

and $\tilde{\gamma}=\gamma \sqrt{\ln 2}$, where $\gamma_{1}, \gamma_{2}$, and $\gamma_{j}$ are the pump, Stokes, and Raman half linewidths, respectively.

The summations $\mathrm{j}$ and $\mathrm{k}$ are over the rotational Raman transitions (i.e. from an $\mathrm{i} \rightarrow \mathrm{f}$ transition) of the vibrat:onal bands of species $s$. The $s$ and $t$ summations are over the species with resc nances contributing to the spectral region of interest. The line shape $F\left(\omega_{4}\right)$ is given by

$$
F\left(\omega_{4}\right)=\int I_{1}\left(\omega_{1}\right) I_{3}\left(\omega_{3}\right) I_{2}\left(\omega_{1}+\omega_{3}-\omega_{4}\right) d \omega_{1} d \omega_{3}
$$

The width of Raman lines are functions of pressure, temperature, and rotational quantum number. Collisional narrowing occurs when the interference between adjacent lines overlap because of line broadening. In the collisional narrowing region, Equation 2 needs to be modified to include the effects of collisional narrowing. Following the treatment of Koszykowski et al with eigenvalue eigenvector approach, $\chi^{R}$ can be simplified to ${ }^{16}$

$$
\chi^{R}=\sum_{j} \frac{a_{j}^{\prime}}{\lambda_{j}^{\prime}-\left(w_{1}-w_{2}\right)+i \lambda_{j}^{\prime \prime}}
$$

where $\lambda_{j}=\lambda_{j}^{\prime}+i \lambda_{j}^{\prime \prime}$ is a complex eigenvalue of the G-matrix; $a_{j}^{\prime}$ is, in general, a complex weight factor. The matrix $G$ is defined as

$$
G_{j k}=\delta_{j k}\left(\Omega_{j}-\left(\omega_{1}-\omega_{2}\right)+\Delta_{j}-i \gamma_{j}\right)+i\left(1-\delta_{j k}\right) \gamma_{j k}
$$

and $\delta_{\mathrm{jk}}$ is a Kronecker delta function. To calculate the convoluted CARS spectrum, Equations 3-5 are still valid for the collisional narrowing CARS spectrum. However, $a_{j}$ 
needs to be replaced with $\mathrm{a}_{\mathrm{j}}^{\prime}$ which is evaluated from the G-matrix approximation; $\Omega_{\mathrm{j}}$ needs to be replaced with the real part of the eigenvalue of the G-matrix, and $\gamma_{j}$ needs to be replaced with the imaginary part of the eigenvalue of the G-matrix.

Computer codes to calculate the CARS spectra of molecular species common to combustion vis. $\mathrm{N}_{2}$ and $\mathrm{CO}$, have been developed and published. ${ }^{18}$ All molecular constants and linewidths used to calculate the $\mathrm{N}_{2}$ and CO CARS spectra used in our CARS measurements are listed and referenced in that work. 


\section{DATA REDUC'TION}

CARS reduction is performed by comparing the spectral shape of a CARS spectrum to calculated CARS spectra. As noted in Equations 4 and 5, the temperature dependence is contained in the $\mathrm{a}_{\mathrm{sj}}$ factor and cannot be separated from the summations. Unless a very fast computer is available, this requires that a library of spectra as a function if temperature be precalculated to serve as a table for the fitting function used in the analysis program. We find that calculating the temperatures in $50 \mathrm{~K}$ intervals and using linear interpolation between the calculated values introduces insignificant error for the spectral resolution used here. The mole fraction parameters $C_{s}$ are separated from the resonance summations allowing them to become parameters in the fitting function, independent of the temperature table. This separation was first used by Hall and Boedeker. ${ }^{17}$ The spectral characteristics of the Stokes radiation are entirely described by the $\mathrm{F}_{4}\left(\omega_{4}\right)$ factor, also independent of the temperature table. This allows a function of nearly arbitrary form to represent the Stokes spectral profile and permits properties of this function to be used as fitting variables. Since the Stokes center frequency tends to shift during the measurement, allowing the Stokes center frequency to vary during analysis improves the accuracy of the inferred values of the temperature and concentration. A weighted average is used to calculate $\chi^{\text {nr }}$ based on the expected mole fractions and nonresonant susceptibilities measured by Rado ${ }^{19}$ and Lundeen $e$ t al. ${ }^{20}$ as modified by Farrow and Rahn. ${ }^{21}$

A fitting program has been developed to analyze CARS data. Since particles in a gas stream can contribute a nonresonant signal through the phenomena of particle-induced laser breakdown, the fitting program accounts for the possible hackground from a particleinduced breakdown. The program employs a nonlinear, least squares fitting subroutine and is based on the Marquardt algorithm. The following parameters can be varied during analysis: temperature, mole fraction parameter, calibration frequency, dispersion, baseline count level, normalization factor, Stokes laser center frequency, Gaussian background (for 
particle effects), and Stokes laser bandwidth. The program user also has the option of fixing any of the above fitting parameters. To reduce the analysis time, a library of CARS spectra should be pregenerated between 300 and $3000 \mathrm{~K}$ in increments of $50 \mathrm{~K}$. Data analysis is performed by fitting the experimental spectrum with the CARS library spectrum. The calculated spectra need to be convoluted with an instrument function before they are compared with the data. The instrument profile used in the data analysis was extracted from a room temperature CARS spectrum recorded before the measurement. Theoretical studies show that the accuracy of temperature or species concentration measurement with CARS spectral shape fitting depends on the accuracy of the theoretical model, the signal/ noise ratio of CARS data, and the knowledge of the experimental parameters such as linewidth of lasers, laser profiles, slit function etc.

CARS temperature measurement is generally performed with the major species in the medium. Since the spectral shape will depend mainly on temperature for high concentration species, temperature will be the main fitting parameter. The accuracy of the temperature measurement, then, can be improved with less parameter in the fit. In some concentration ranges, the spectral shape can be sensitive to both temperature and species concentration, then temperature and concentration can both be extracted from the CARS data.

Concentration measurement by fitting the shape of the CARS spectrum is limited to certain concentration ranges because the shape of CARS spectrum is sensitive to concentration only when the species concentration is close to the point where the resonant and background susceptibilities become comparable. In general, the spectral shape is a sensitive indicator of concentration in the range from approximately 1 to $30 \%$. Since the CARS analysis program can only exlaact the mole fraction parameter, which is the ratio of species concentration to the nonresonant susceptibility, the accuracy of concentration measurement depends on the knowledge of the nomresonant susceptibility. The nonresonant 
susceptibility can generally be calculated using the approximate concentration of the species in the medium. If the nonresonant background cannot be accurately predicted, the concentration measurement with CARS becomes impractical. 


\section{PHASE MATCHING}

The CARS signal is at maximum if the phase matching condition is met (i.e. $\Delta \mathrm{k}=0$ )). The phase matching condition can be achieved by properly aligning the geometries of the pump and Stokes laser beams. There are different methods by which to achieve the phase matching condition. The most commonly used geometries are shown in Figure 4 . The simplest technique is collinear CARS, in which the pump and Stokes beams aligned on top of each other travel collinearly toward the test medium as shown in Figure 4a. The antiStokes beam is generated along the same direction of the laser beams.

USED CARS phase matching geometry is quite similar to collinear phase matching, in the sense of laser beam alignment. The Stokes beam is aligned inside the donut-shaped pump beam as shown in Figure $4 \mathrm{~b}$. The donut-shaped pump beam is transformed into the Gaussian heam in the focal volume where the pump and Stokes beams overlap. The emerging geometry is also easy to align, and it provides better spatial resolution than does the collinear phase matching geometry.

In the planar BOXCARS geometry, shown in Figure 4c, a pump and Stokes beam overlap collinearly to each other and parallel to the second pump beam. The CARS signal generated at the crossing point of the three laser beoms tuavels collinearly with the second pump beam. The folded BOXCARS phase matching is an improved form of the planar BOXCARS, in which three laser heams are independent. If all three beams lie on the circumference of a circle, two pump beams are at the two ends of a diameter, and the Stokes beam is at the center of the halt-circle arc. The position of the anti-Stokes beams is opposite the Stokes laser beam and spatially separated from the pump and Stokes laser beams. The filtering of the CARS signal from the high-intensity laser beam in folded BOXCARS is easier than in other phase matching geometries. 
(a) Collureaı

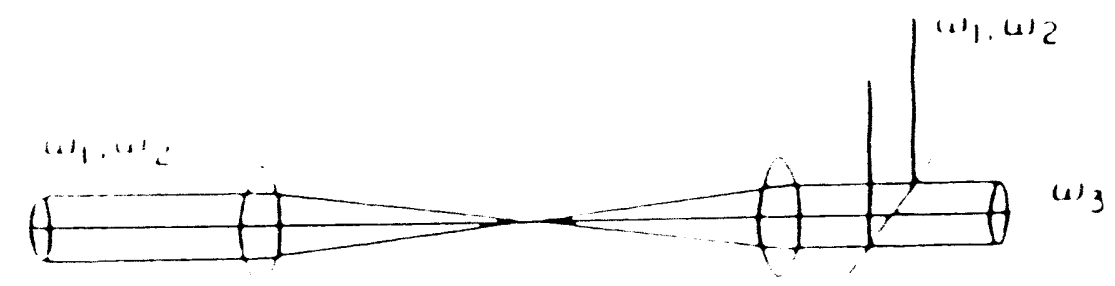

(b) IISED CARS

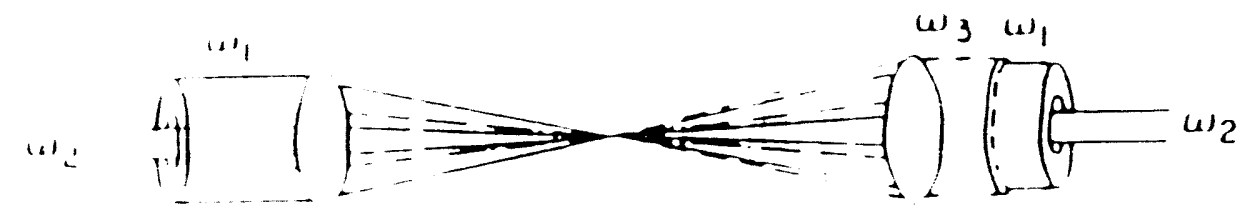

(c) Planar BOXCARS

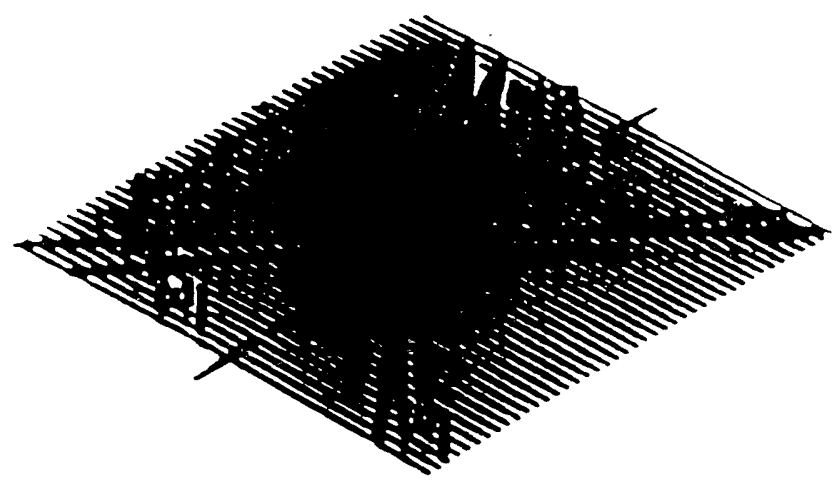

(d) Folded BOXCARS

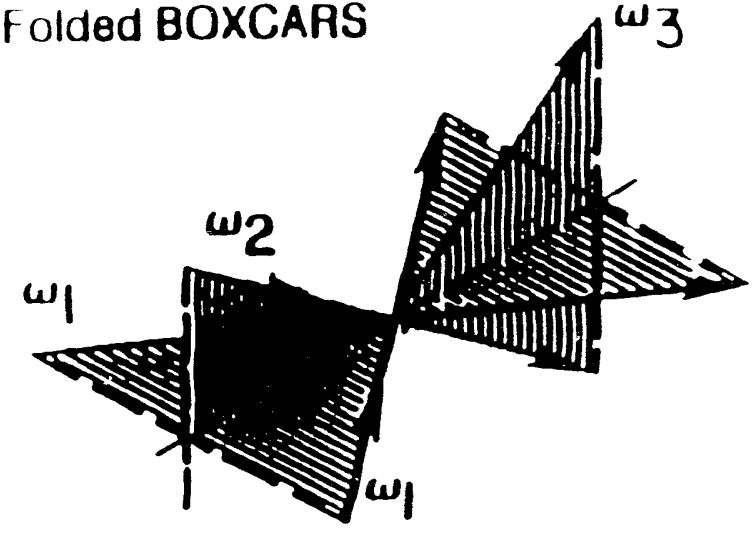


The optical alignment of the laser beams in BOXCARS geometry is more difficult than collinear and USED CARS; however, the spatial resolution is better with BOXCARS. The alignment in BOXCARS geometry is more sensitive to turbulence in the air and in the gas stream than are the collinear and USED CARS geometries. 


\section{SYSTEM DESCRIPTION}

This mobile CARS instrument is the compact, modified version of the CARS setup reported earlier ${ }^{22}$ - shown in Figure 5. It is designed for combustion diagnostics in a variety of test facilities. The complete system is assembled in a trailer that can be transported to a desired test facility. The main units of the instrument are a transmitter, a receiver, a traversing lens system, and a data acquisition/analysis system, as shown in Figure 5. Each item of the instrument is described below.

\section{Transmitter}

The transmitter is used to manipulate the laser beams into a particular phase matching geometry and to transmit them to the test medium. It consists of a custom-built, Qswitched Nd:YAG laser-Spectra-Physics DCR-4D(10)-that produces a pulse of $1.2 \mathrm{~J}$ of energy at $1.06 \mu \mathrm{m}$. This IR bearn is passed through a temperature-stabilized frequency doubler (HG-2A) to produce a green beam at $532 \mathrm{~nm}$ of $450 \mathrm{~mJ}$ pulse energy. The residual IR beam and green laser beam are separated using a Pellin Brocca prism (PB-2) and a $180^{\circ}$ folding prism (G002-(0)2) from Spectra-Physics. The residual IR beam is passed through the second doubler (HG-2A) to generate a secondary $532 \mathrm{~nm}$ laser beam. The secondary green beam is separated from the IR beam with a dichroic mirror that transmits most of the remaining IR beam to a beam dump and reflects the $532 \mathrm{~nm}$ green beam. Any collinear IR radiation with the secondary green beam will then be absorbed by a Schott KGl $2 \mathrm{~mm}$ thick IR filter: This secondary green beam pumps the oscillator of the broadband dye laser.

A compact and flexible dye laser similar to the one described in reference 10 was designed. The same dye cell is used for the oscillator and the amplifier. The secondary green beam is focused into one side of the dye cell to pump the dye laser oscillator. The dye laser cavity mirrors are mounted on a special 1 " mirror mount that has a $5 \mathrm{~mm}$ diameter hole located $20 \mathrm{~mm}$ away from the center of the mount. 


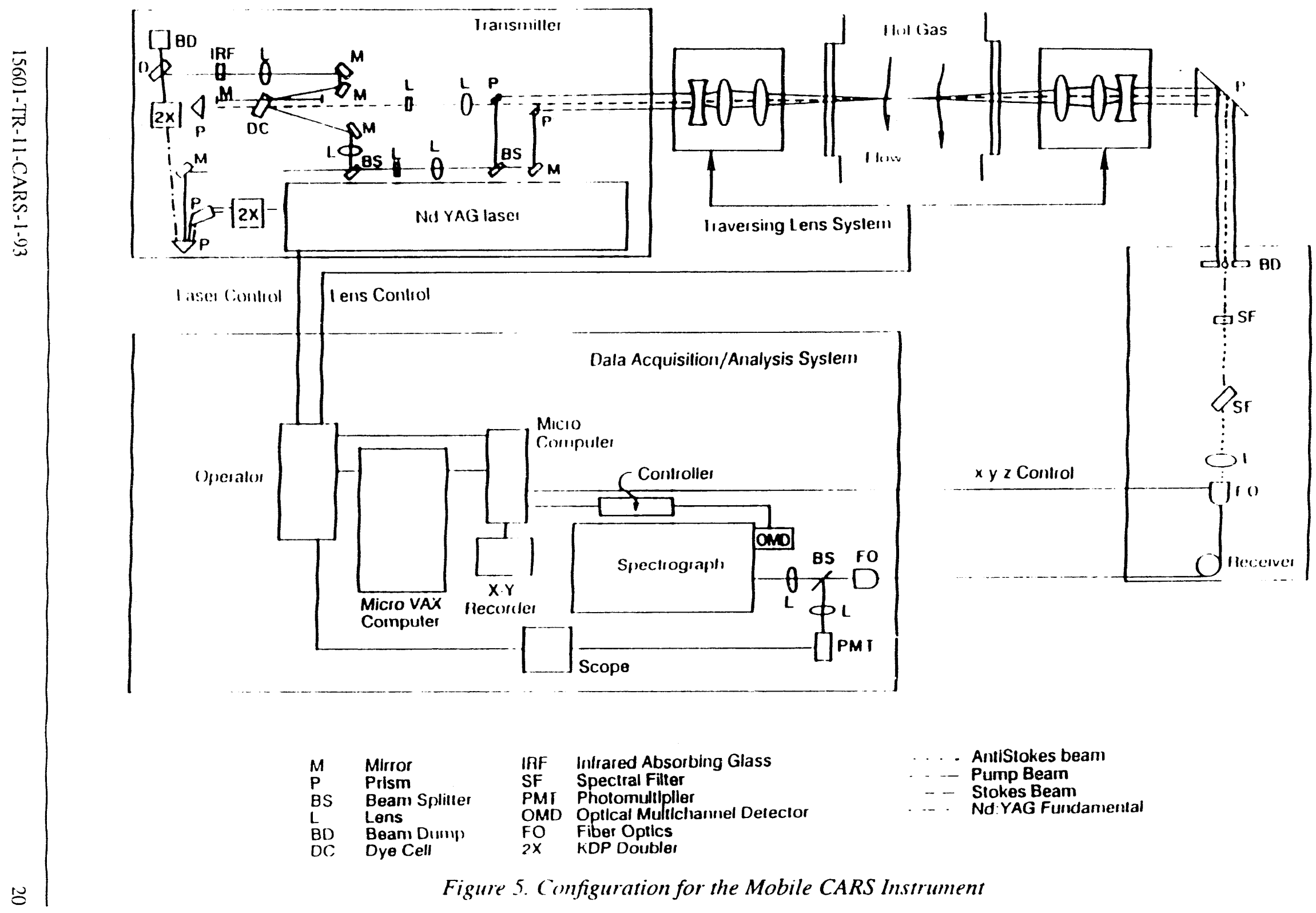




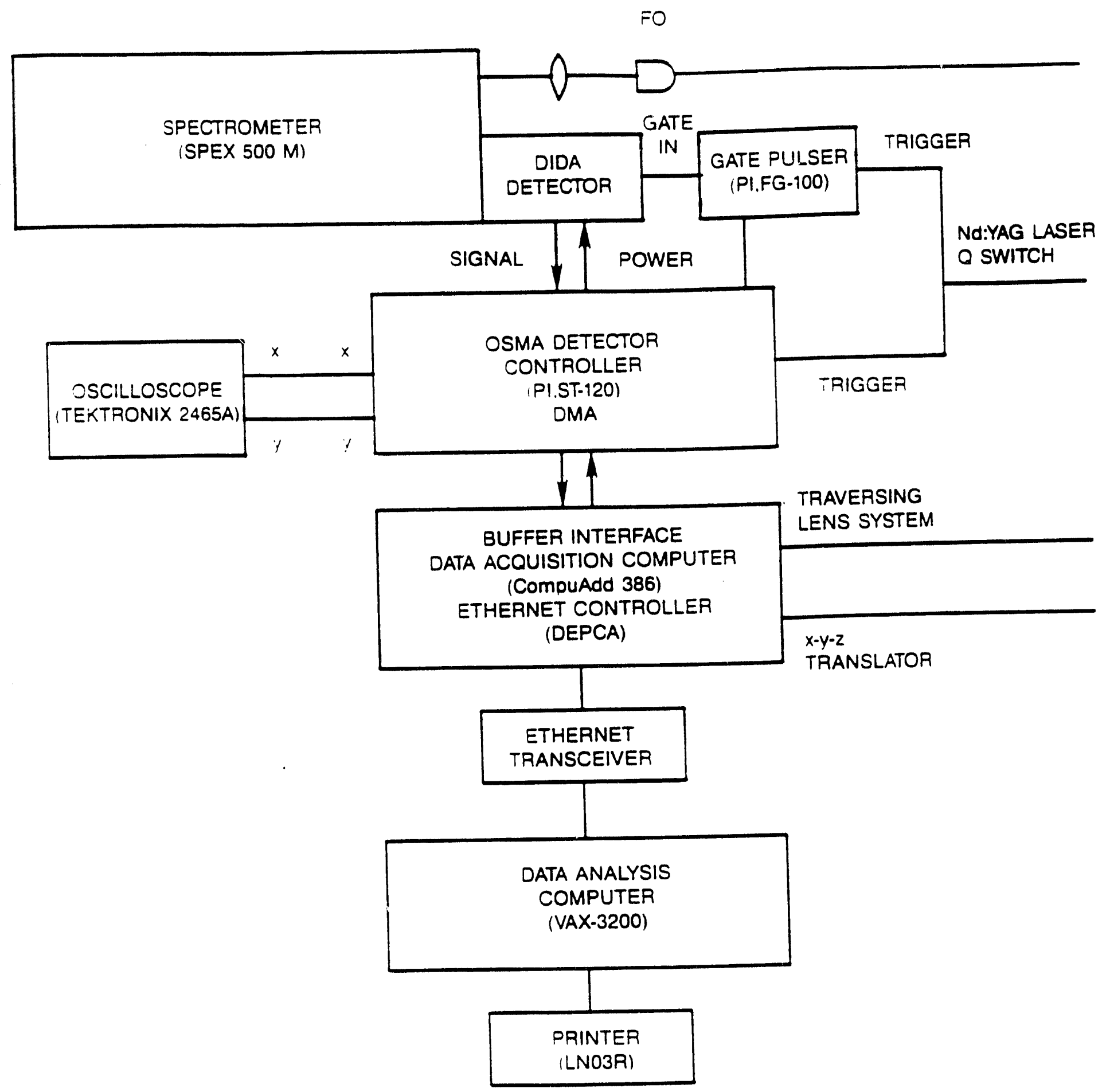

Figure 6. A block diagram of the CARS data acquisition/analysis sustem 
The output of the oscillator is folded back by a rooftop prism and passes through the hole on the mirror mount and the dye cell. One third of the primary green beam is focused into the dye cell to amplify the output of the oscillator. The output power of the dye laser is 45 mJ per pulse with a $9 \times 10^{5} \mathrm{M} / 1 \mathrm{Rh} 640$ dye solution.

The remainder of the primary green beam is expanded and then divided into two parallel beams of equal intensity that are needed for BOXCARS phase matching geometry. The dye laser output is also expanded with a Galilean telescope. Then the green beams (pump beams) and the Stokes beam are aligned parallel to each other. The final configuration of the beams depends on the phase matching chosen, e.g., collinear or BOXCARS. Both the telescopes in the pump lasers and in the dye laser (Stokes beam) can be adjusted to allow the laser beams to focus at their crossing point.

The transmitter optics and laser head are mounted on a $4^{\prime} \times 6^{\prime} \times 8^{\prime \prime}$ optical table. To make more efficient use of space available in the mobile CARS laboratory, the leg positions of the optical table are rearranged so that the cooling unit (NESLAB CFT-75) and the redesigned, custom-built power supply of the Nd:YAG laser can fit under the table. The cooling unit, during hot weather, is kept in an air conditioned wooden box under the mobile electronic laboratory to maintain the temperature of the mobile CARS laboratory at an efficient $72^{\circ} \mathrm{F}$. An aluminum plate $3^{\prime} \times 4^{\prime} \times 1 / 2^{\prime \prime}$ was mounted above the optics table to hold the dye circulation pump, the laser power meter, and any extra optics needed in the field. The transmitter optics table also has essential receiver optics to test CARS laser beam alignment in the laboratory. An argon-filled, stainless steel cell (3" inner diameter and $1 / 4 "$ wall thickness) is also mounted on the transmitter table to produce nonresonant CARS to monitor the dye profile.

\section{Traversing Lens System}

The traversing lens system is designed to focus the laser beams into the test medium. The lens system consists of one diverging lens $F_{1}$ and two converging lenses $F_{2}$ and $F_{3}$. 
The focal lengths of these lenses are $f_{1}, f_{2}$, and $f_{3}$, respectively. Lenses $F_{1}$ and $F_{2}$ form a Galilean telescope and lens $F_{3}$ is the field lens. The positions of $F_{2}$ and $F_{3}$ are fixed, and the separation between them is equal to the sum of their focal lengths $\left(f_{2}+f_{3}\right)$. The separation between lenses $F_{1}$ and $F_{2}$ defiries the focal point position of the lens system. A small displacement in the position of the $F_{1}$ lens provides a large change in the position of the focal point. The details of this system are given in reference 23 .

The lens system focuses the laser beams into the test region where the CARS signal is to be generated. The CARS signal, along with the laser beams in the forward direction, are recollimated through another similar traversing lens system and directed to the receiver. Since this is a three-lens system, it uses more space and reduces the signal. In most of the applications described in this topical report, a single lens traversing system has been used for focusing and collimating the CARS laser beam and signal.

\section{Receiver and Detection System}

The receiver was assembled on an 18 " $\times 24$ " aluminum plate mounted close to the exit port of the test facility. It contains the dichroic mirror and beam blocks that separate the CARS signal from high intensity laser beams. The CARS signal is then coupled to the optical fiber mounted on a computer-controlled $x-y-z$ translator with suitable coupling optics for maximum transfer of the CARS signal. An optical fiber of $200 \mu \mathrm{m}$ core diameter and 0.12 numerical aperture (NA) from the Fiberguide Industries is used to bring the CARS signal from the receiver to the detection system. A $100 \mu \mathrm{m}, 0.12 \mathrm{NA}$ optical fiber is also available for the field measurement. However, in a vibrating combustion facility, it is easier to couple the CARS signal with a $200 \mu \mathrm{m}$ optical fiber than with a $100 \mu \mathrm{m}$. The detection system includes a SPEX 500M spectrograph with f-number of 4 and a double intensified diode array (DIDA) detector from Princetcon Instruments Inc. mounted on the exit of the spectrograph. The optical fiber coupler is specially designed for maximum 
transfer of the CARS signal from the fiber to the spectrograph. In some cases, the exit end of the optical fiber will act as an entrance slit of the spectrograph by direct coupling of the optical fiber with the spectrograph. The detection sensitivity can be enhanced five times with direct coupling method as compared to the CARS signal obtained with a $60 \mu \mathrm{m}$ spectrograph slit width. The f-number of the optical fiber (3.8) is smaller than that of the spectrograph so that the grating is not over-filled with CARS signal which eliminates the coupling losses.

A glass slide is placed in front of the optical fiber coupling optics on the receiver as a beam splitter to send a part of the CARS signal to the PMT tube. A narrow band interference filter is mounted in front of the PMT to allow only the CARS signal to transmit; the PMT output is monitored by a Tektronics 2465A oscilloscope. This system is used to optimize the alignment of the pump and stokes laser beams in the interaction region and the coupling of the CARS signal with the optical fiber.

\section{Data Acquisition/ Analysis System}

The schematic diagram of the data acquisition/analysis system is shown in Figure 6. An IBM-compatible computer (Dell 486) is interfaced to the detector-controller (Princeton Instruments model ST-120) which digitizes and transmits the acquired data to be manipulated and stored by the computer via direct memory access (DMA). The ST-120 controller provides power, thermostating, control and signal timing to the detector head. Its flexible timing mode can coordinate data acquisition with the experiment. The $x-y-z$ axis output of the controller is for real time data monitoring using an oscilloscope. The ST120 software package developed by Princeton Instruments controls data acquisition. It provides near real-time graphics display and real-time data storage on a hard disk. A stepping motor control program has been developed and integrated into the ST-120 software for moving the lens traverse system and the optical fiber position during the experiment. The data collected on the Dell computer hard disk is transferred to a VAX-3200 worksta- 
tion for analysis. The Dell computer and the VAX-3200 workstation are integrated into a network using an Ethernet controller (DEPCA) which provides direct information access and sharing through the network.

The laser-induced breakdown has been considered a problem for accurate CARS measurements in a coal-fired combustor, ${ }^{6}$ because the huge background suppresses the resonant CARS signal. A data selection program was developed in the Dell computer to select the data with less laser-induced breakdown. Only the data with a reasonable background is selected and transferred to the VAX-3200 computer for analysis. In some measurements the collinear phasematching geometry is used. Since the transmitter may be $30^{\prime}$ to $100^{\prime}$ away from the measurement point, depending on the test facility, the collinear CARS signal may be generated outside the measuring volume if the operators are not careful. A computer routine was developed to pre-examine the data by comparing the bandwidth of the cold and hot bands before further processing. The CARS data analysis software was developed on the VAX-3200 computer. It can extract the instrument response function from a room-temperature CARS spectrum; then the extracted parameters are supplied for CARS data analysis. The fitted results can be stored in a file or output to a printer (DEC LNO3R).

\section{Mobile CARS Laboratory And Beam Delivery System}

An electronic mobile laboratory was designed and constructed from a 43' long trailer to contain the various combustion diagnostic instruments for field application, as shown in Figure 7. A small room $\left(7-1 / 2^{\prime} \times 11-1 / 2^{\prime}\right)$ at the back of the trailer contains the mobile CARS system. The trailer's temperature is controlled $\left(T=72 \pm 2^{\circ} \mathrm{F}\right)$ to protect the laser head and to reduce thermally-induced optical misalignment in the transmitter. In field applications, the trailer is parked $30^{\prime}$ to $100^{\prime}$ from the test facility.

The beam delivery system sends the laser beams emerging from the transmitter to the test media using a series of right angle tuming prisms. The laser beams are sent out 


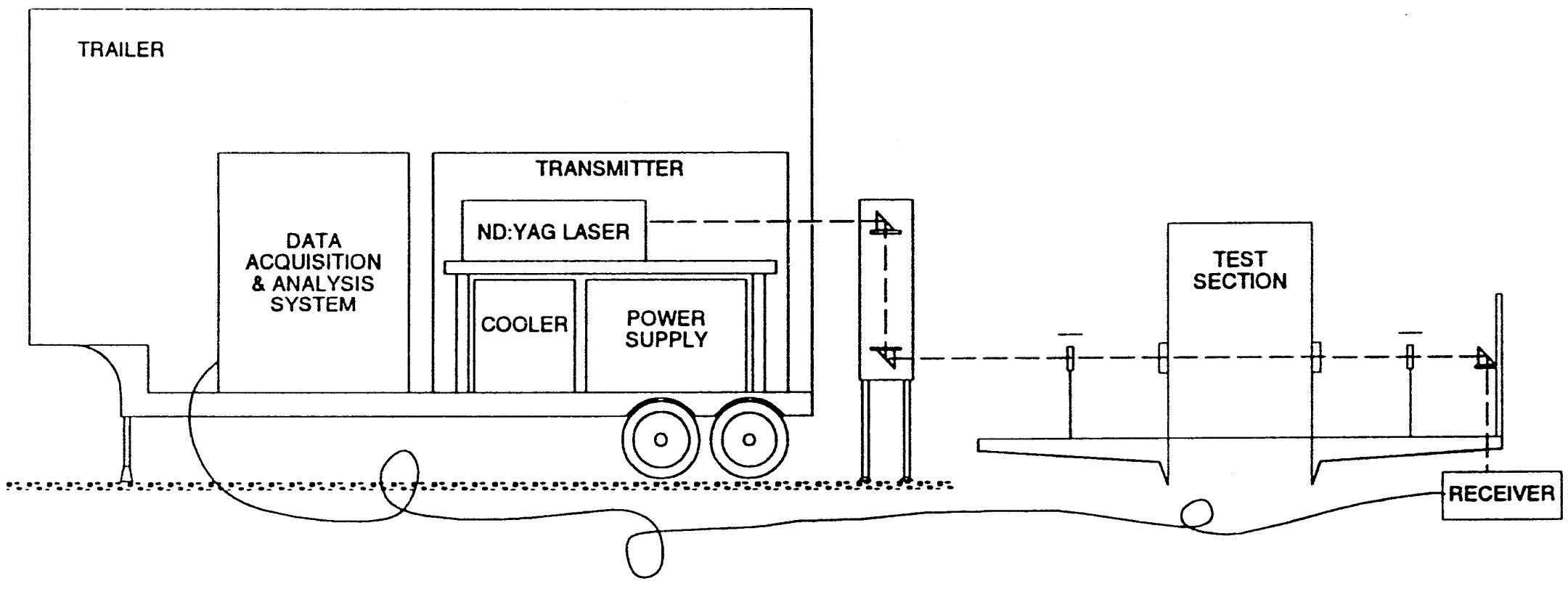

Figure 7. Arrangement of the Mobile CARS system components 
through a hole in the back of the trailer. Two turning prisms are mounted on an iron stand, and may be raised or lowered to align the beams with the optical port. The beam delivery system can be modified easily to fit the needs of various ports or test facilities. 


\section{OPERATION}

The DIAL mobile electronic laboratory is moved to the desired test facility and parked at a predetermined safe location. Four jacks are used to lift the laboratory slightly, leveling it and reducing its direct contact with the ground through its tires, decreasing the vibration in the CARS laboratory. Leveling the trailer keeps the instruments close to level, but there is a provision for leveling the optics table of the transmitter and detection system should it be necessary.

The major instruments and some of the optics of the mobile CARS system, packed before the trailer is transported to the test facility are unpacked upon arrival. The Nd:YAG laser, monochromator and computers are unpacked and tested for proper operation. The Nd:YAG laser is tweaked for optimum power, and various optics of the transmitter are aligned for the desired phase matching geometry. To check the alignment, the laser beams are manipulated the same distance to a measuring point inside the laboratory with the prisms attached to the ceiling of the CARS laboratory. To optimize the CARS signal, the crossing of the pump and Stokes laser beams at focal volume is checked with a knife edge mounted on an $x-y-z$ translator. This test is performed with the field lens in the air inside the mobile CARS laboratory.

A HeNe laser installed in the transmitter is used to align the dye laser, beam delivery optics, and the lens traverse system. The HeNe laser beam is passed through a hole in the wall of the trailer and passed through the center of the right angle turning prism; then, it is manipulated through the port windows. The positioning of the HeNe laser beam defines the axis of the CARS laser beams. The position of the three-legged, iron stand behind the trailer is adjusted so that the HeNe laser beams passes though at roughly the center of the ports. The final adjustments are made with prisms mounted on the stand to keep the laser beam at the center of the port window. The axis of the lens traverse system is then aligned with the HeNe laser beam. Two pin holes mounted on the two ends of the traverse system 
are used to align the axis of the lens traverse system.

After all the optics in the beam delivery system and receiver are aligned with the HeNe laser, CARS laser heams aligned for a particular phase matching geometry are then sent to the beam delivery system. These laser beams are manipulated and aligned to the optical port of the test facility and focused inside the gas stream. The CARS signal, on the receiver side, is coupled to the optical fiber mounted on the $x-y-z$ translator. The operator can monitor the CARS signal on the oscilloscope with the DIDA detector. The position of the optical fiber is tweaked with the Dell 486 computer to optimize the CARS signal. As soon as CARS spectra is recorded at a point, the focal point is moved to the next measuring point. The CARS signal can be optimized with the optical fiber translator before further recording. 


\section{TEMPERATURE AND CONCENTRATION MEASUREMENT}

\section{Flame Measurement}

Temperature measurement of a $\mathrm{CH}_{4} /$ air llame at different stoichiometry has been performed with the mobile CARS instrument. The details of these measurements are . described in reference 24 . In brief, the mobile CARS laser beams were aligned with a $\mathrm{CH}_{4}$ /air flame as shown in Figure 5. $\mathrm{N}_{2}$ CARS spectra were recorded at different points across the flame. These spectra were fit with a library of computer-simulated spectra to infer the temperature. $\mathrm{CO}$ and $\mathrm{H}_{2}$ concentration measurement in a $\mathrm{CH}_{4}$ / air flame at different stoichiometry and $\mathrm{C}($ ) injection rate has also been performed (as described in reference 25).

\section{DIAL/MSU Test Stand Measurement}

Temperature and CO concentration profile measurements across the DIAL test stand have been performed. ${ }^{25.26}$ Temperature measurement has been performed with $\mathrm{N}_{2}$ CARS spectra recorded with an $\mathrm{N}_{2}$ CARS dye laser. Simultaneous measurement of temperature and $\mathrm{CO}$ concentration have been performed by recording simultaneous $\mathrm{N}_{2}$-CO CARS spectra with a single dye laser. The minimum delection limit of $\mathrm{CO}$ concentration is $\sim 1 \%$ in a clean gas stream; the limit increases to $3-5 \%$ in a particle-laden gas stream. This sensitivity limit and the accuracy of the concentration measurement depend on the eoal particle concentration in the gas stream. CARS inferred temperature with $\mathrm{N}_{2}$-CO CARS spectra is in agreement with the $\mathrm{N}_{2}$ CARS measurement.

\section{CFFF Diffuser Measurements}

A series of experiments was performed with a premixed $\mathrm{CH}_{4} /$ air flame and with the DIAL test stand to evaluate the diflerent phase matching geometries for the field measurements. ${ }^{27}$ Various focusing lenses were also tested to compare the CARS signal and interac- 
tion length with various phase matching techniques. Although the collinear CARS gives the highest signal strength, is easiest to align, and is less sensitive to air turbulence and the vibrations of the mobile CARS instrument and combustor, collinear CARS is not suitable for temperature measurements at the CFFF diffuser because of poor spatial resolution with the long focal length field lens $(109 \mathrm{~cm})$ needed for a large gas stream $(76 \mathrm{~cm}){ }^{28,29}$ The folded BOXCARS phase matching technique provides better spatial resolution $(\sim 10 \mathrm{~mm})$ with a $109 \mathrm{~cm}$ focal length lens. The spatial resolution is defined as the length in which $90 \%$ of the CARS signal is generated. Therefore, the folded BOXCARS phase matching technique is the first choice to measure temperature and the temperature profile in the CFFF.

In flame and DIAL test stand measurement, the DIDA detector is generally operated in continuous mode: the detector is continuously scanned, and the information is stored in the computer. However, the high luminosity from the coal-fired combustor, caused by the emission from the hot coal particles and the broad atomic emission from the potassium seed used in MHD, produces a huge background on the CARS signal; it is impossible to subtract this background while using continuous mode. To reduce the amount of unwanted emissions entering the detector, the DIDA detector is operated in gated mode. An adjustable aperture was mounted on the receiver table to further eliminate the emission from the gas stream. By properly adjusting the gate pulse delay and pulse width with respect to the CARS signal, good quality CARS data can then be recorded. The CARS signal recorded by the DIDA detector generally has $1(0)-2()() \mathrm{ns}$ delay between the laser pulse and the gate's opening. This delay depends on the distance of the test gas stream and the length of the optical fiber used to bring the CARS signal to the detection system. A typical $200 \mathrm{~ns}$ gate pulse width and 100 ns delay between the rising edge of the pulse from the pulser with CARS pulse were used in CFFF measurement. The sensitivity of the detector in gate mode is $40 \%$ less than when in continuous mode. In clean flame measurement, the free- 
running, continuous mode is more uselul.

Temperature measurements were pertormed at dilferent points across the gas stream by moving the focusing lens. The CARS spectrum recorded across the gas stream has two extra bands at the top of the $\mathrm{N}_{2}$ CARS hot band $(v=2 \rightarrow 3)$ which have not been seen in either a $\mathrm{CH}_{4}$ /air flame or the DIAL/MSU test stand. ${ }^{29}$ A typical spectrum recorded in the CFFF diffuser is shown in Figure 8. The new feature is more pronounced near the center of the gas stream, and it is not related to $\mathrm{N}_{2}$ CARS. Regardless of the origin of the feature, it is believed that meaningful temperatures can be obtained from the analysis of the data. This spectrum has been fit with the compuler simulated spectra by excluding the data points which contain the new features. The inferred temperature from the fitting was found to be $2684.9 \mathrm{~K}$. The complete spectrum including the new leature was also fit with computer simulated spectrum, and the inferred temperature was found to be $2686.6 \mathrm{~K}$. The inferred temperature with and without the new feature in the fitting are quite close to each other and are in agreement with the temperature measured from Sodium Line Reversal (SLR) at a port $\sim 2$ upstream from the CARS port. A temperature profile was obtained by analyzing the CARS data across the diffuser by excluding the new feature from the spectra. Results of the analysis are shown in Figure 9. To avoid window damage from the high temperature and slag deposits in the port, the optical ports were purged with dry nitrogen during the measurements. Since spectra recorded close to the boundary of the gas stream or inside the port look much colder than expected because of the cold nitrogen contribution from the $\mathrm{N}_{2}$ purge, the data were also collected using an argon purge. 


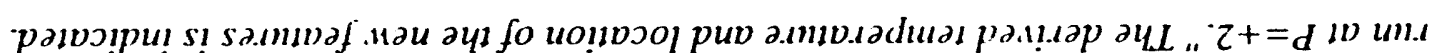

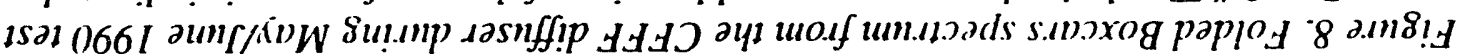

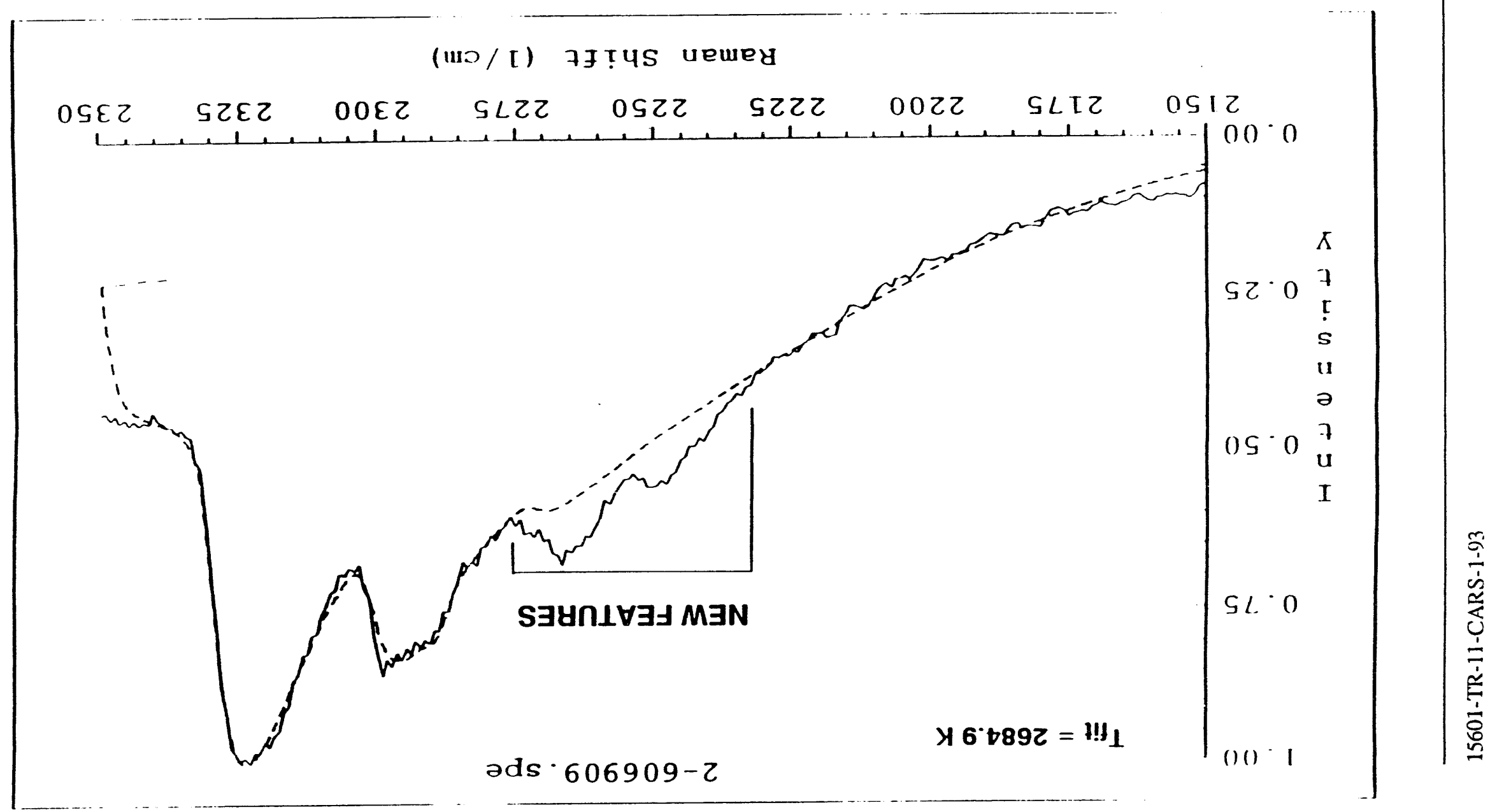


UTSI DIFEUSER CARS DATA

DATE : JUNE 6, 1990

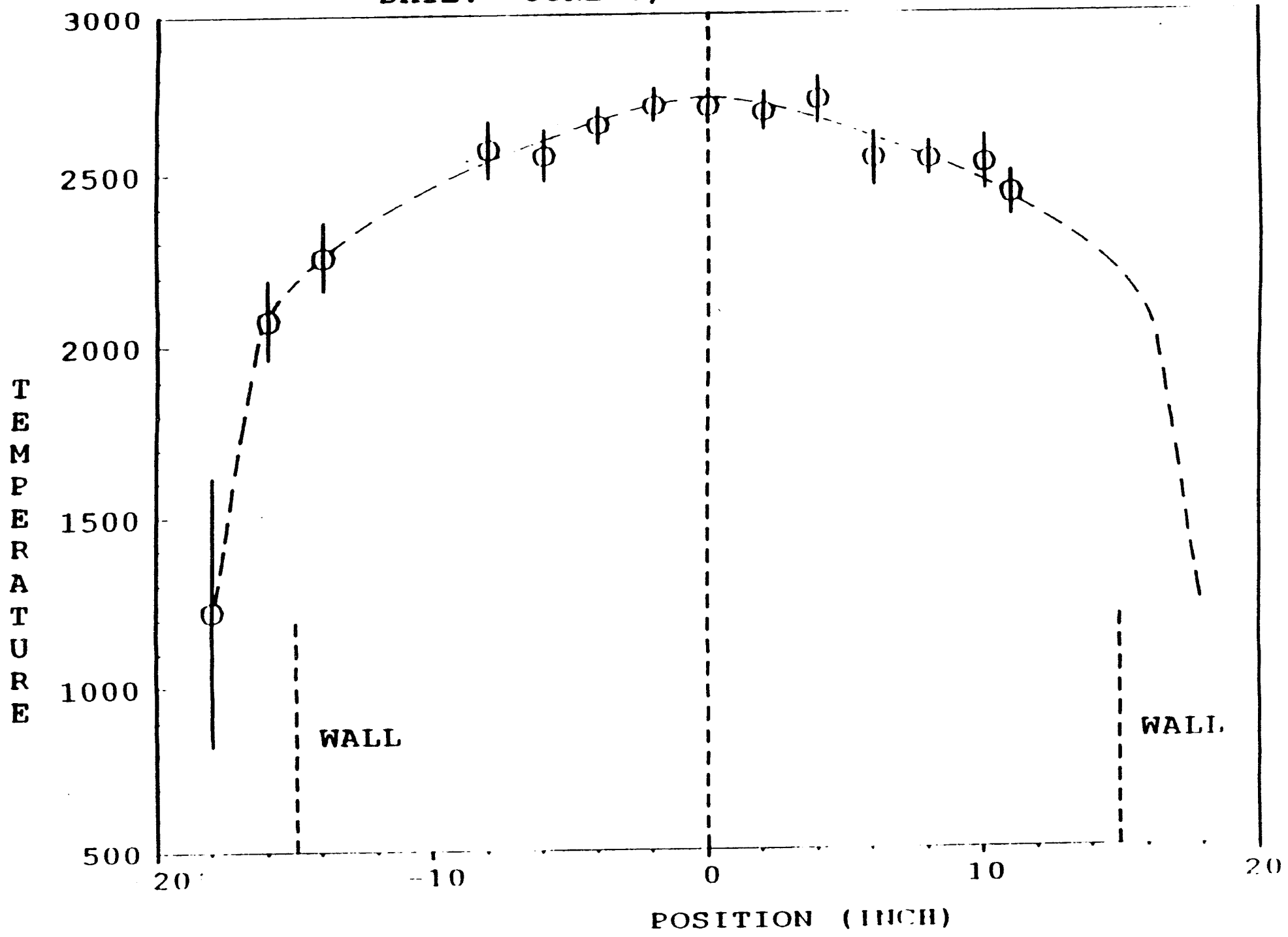

Figure 9. Temperature profile of the CFFF diffuser during the LMF4-T test. 


\section{CFFF Aerodynamic Duct Temperature Measurements}

The mobile CARS system was aligned at the CFFF aerodynamic duct during the October 1992 LMF5-G test run to measure the temperature profile. The aerodynamic duct CARS data showed interference from particle-induced laser breakdown effects. This breakdown occurs because of the high laser power and high particle density during these measurements. This breakdown sometimes generates atomic emission lines which can interfere with the CARS signal. However, the signal to noise ratio(S/N) of the data are good enough for data reduction. Figure 10 shows a typical CARS spectra (upper curve) taken at 0.25 " from the center of the gas stream on the east side of the duct. The dip in the observed spectrum near $2320 \mathrm{~cm}^{-1}$ is due to $C_{2}$ absorption and occurs at the $0-1 \mathrm{~N}_{2}$ CARS band. The temperature was extracted by fitting the spectral regions that are not affected by $\mathrm{C}_{2}$ absorption. The lower curve of Figure 10 is the difference between the calculated and observed spectra. Because of the substoichiometric conditions, the concentrations of various hydrocarbon molecular species, which contributed to the nonresonance susceptibility, are not known accurately. To take into account the variation of the nonresonant susceptibility and the $\mathrm{N}_{2}$ concentration to the probe volume, the $C$ parameter (ratio of the $\mathrm{N}_{2}$ mole fraction to the non resonant susceptibility) was floated in the least squares fit. The error in temperature by fitting mainly the hot band and a small part of the cold band was estimated to be about $200 \mathrm{~K}$. 


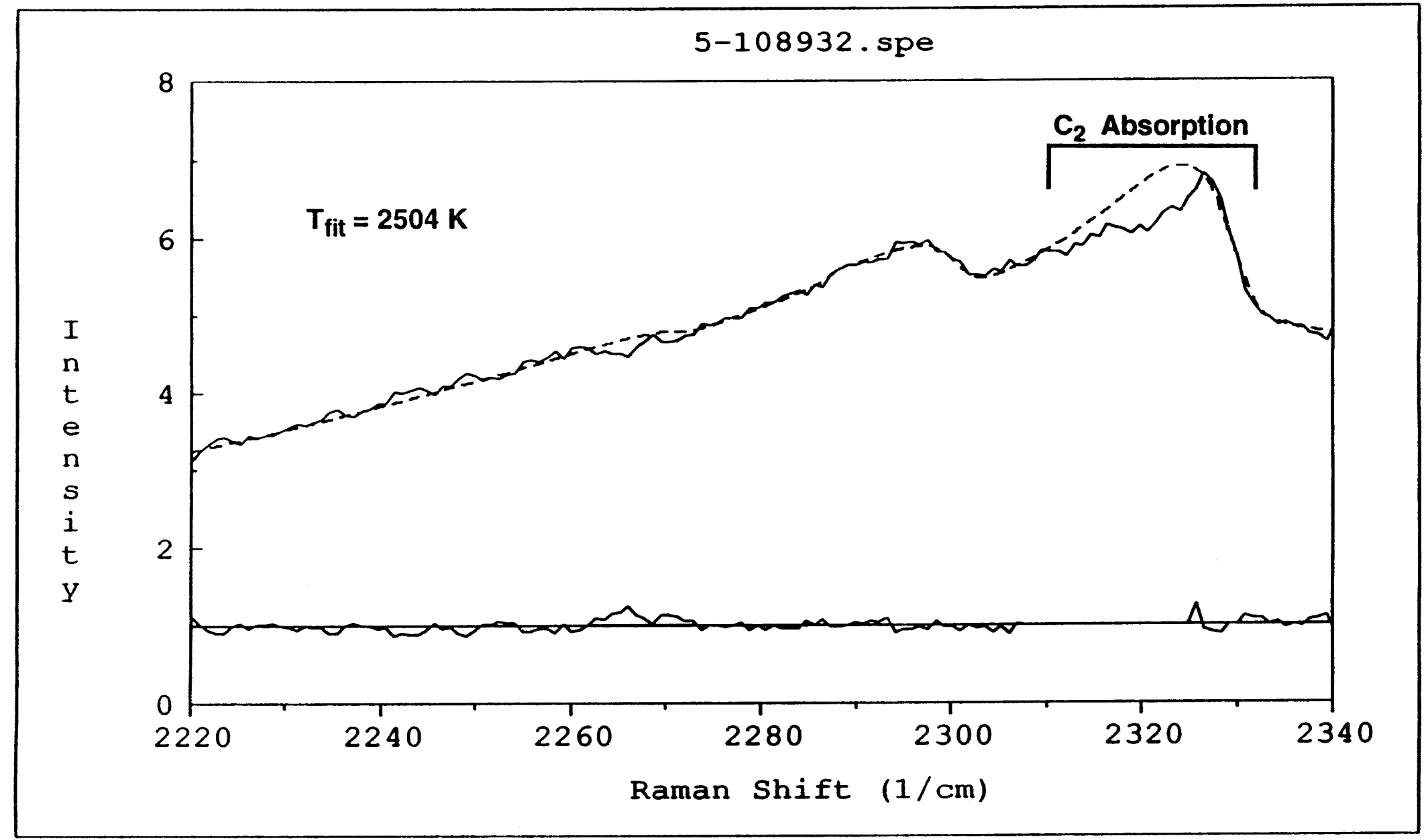

Figure 10. CARS spectrum recorded at the aerodynamic duct during the LMF5-G test run. $\mathrm{P}=0.75 "$ $\mathrm{T}_{\mathrm{fit}}=2504 \mathrm{~K}$. The lower curve is the difference between the calculated and observed spectra 


\section{Problems}

Various unexpected experimental problems that arise in the field and help to design the instrument for other industrial combustion facilities are worth mention. Since the transmitter may be $30^{\prime}-100^{\prime}$ from the test facility to avoid severe beam attenuation and beam steering, it is important to choose a short optical path to the test facility with minimum number of optics in the beam delivery system.

The phase matching geometry for a particular industrial application must be pre-evaluated in order to obtain a precise measurement. ${ }^{26}$ The Collinear CARS phase matching geometry has always been considered as a first choice for field application because of its high signal strength, ease of alignment, and lack of sensitivity to turbulence and vibrations. However, the CARS signal was generated before the focal point while the pump and Stokes beams overlapped. With collinear CARS phase matching geometry, the signal generated outside the tested volume might be appreciable because of the long optical path from the transmitter to the optical port. A series of experiments has been conducted in the DIAL test stand and a $\mathrm{CH}_{4} /$ air flame to evaluate this problem. The optical arrangement was modified to eliminate the contributions of the CARS signal generated outside the optical ports. The details of these measurements are in references 24 and 28 . In brief, a GG495 Schott filter was attached to the entrance port window to eliminate the CARS signal generated before the entrance port window. A 532-nm reflecting dichroic mirrormounted in an aluminum pipe with an argon-purge-was attached to the exit port window to stop the CARS signal generation after the exit window. Argon purge was used at both ports to eliminate the CARS signal generated between the gas stream and the port window. The pump and Stokes laser beams were expanded to $45 \mathrm{~mm}$ and were focused together at their crossing point. This modification made it possible to record the $\mathrm{N}_{2}$-CARS signal generated from the hot gas stream.

It is clear that various problems may occur while making measurements using the 
mobile CARS instrument. However, ihe problems lead us to modify the system for better performance. It is important to select the site to park the Mobile CARS laboratory and the phase matching geometry used for a particular industrial facility, as mentioned above. Potential problems in the field must also be evaluated before making measurements. 


\section{ACKNOWLEDGEMENTS}

The authors would like to thank E.J. Beiting, Aerospace Corporation and John Luthe of DIAL/MSU, who were involved in the beginning of the CARS work, and R.L. Cook DIAL/MSU for their discussions during the measurements. The authors would also like to thank DIAL/MSU professional and technical staff for their help during the development of the mobile CARS instrument. 


\section{REFERENCES}

1. R. J. H. Clark and R. E. Hester, Ed., Advances in Nonlinear Spectroscopy, (John Wiley and Sons, NY, 1988).

2. A. C. Eckbreth, Laser Diagnostic for Combustion Temperature and Species, (Abacus Press, Tunbridge Wells, 1988).

3. S. A. J. Druet and J. P. E. Turan, Prog. Quantum Electron Z, 1 (1981).

4. K. Klick, K. A. Marko, and L. Rimai, Appl. Opt. 20, 1178 (1981).

5. A. Hartford, D. A. Cremers, T. R. Loree, G. P. Quigley, L. J. Radziemski, and D. J. Taylor, Proceedings of the Society of Photo Optical Instrumentation Engineering $411,92(1983)$.

6. E. J. Beiting, Appl. Opt. 25, 1684 (1986).

7. M. Alden and S. Wallin, Appl. Opt. 24, 3434 (1985).

8. R. D. Hancock, P. O. Hedman and S. K. Kramer, Combustion and Flame 87, 77 (1991).

9. A. C. Eckbreth, B. M. Dobbs, J. H. Stufflebeam, and P. A. Tellex, Appl. Opt. 233, 1328 (1984).

10. T. J. Anderson, G. M. Dobbs, and A. C. Eckbreth, Appl. Opt. 25, 4076 (1986).

11. M. A. Yuratich, Mol. Phys. $\underline{38}, 625$ (1979).

12. H. Kataoka, S. Maeda, and C. Hirose, Appl. Spectrosc. 36, 565 (1982).

13. R. E. Teets, Opt. Lett. $\underline{9}, 226$ (1984).

14. D. A. Greenhalgh and R. J. Hall, Opt. Commun. $\underline{57}, 125$ (1986). 
15. F. Y. Yueh and E. J. Beiting, Comput. Phys. Commun. 42, 65 (1986).

16. M. L. Koszykowski, R. L. Farrow, and R. E. Palmer, Opt. Lett. 10, 478 (1985)

17. R. J. Hall and L. R. Boedeker, Appl. Opt. 23, 1340 (1984).

18. J.C. Luthe, E.J. Beiting, and F.Y. Yueh, Comput. Phys. Commun. 42, 73 (1986).

19. W. G. Rado, Appl. Phys. Lett. 11, 123 (1967).

20. T. Lundeen, S. Y. Hou, and J. W. Nibler, J. Chem. Phys. 79, 6301 (1983).

21. R. L. Farrow and L. A. Rahn, J. Opt. Soc. Am. B2, $9(33$ (1985).

22. J. P. Singh and F. Y. Yueh, Appl. Opt. 30, 1967 (1991).

23. A. C. Eckbreth and J. H. Stufflebeam, Appl. Opt. 24, 1405 (1985).

24. J. P. Singh and F. Y. Yueh, Combustion and Flame. 89, 77 (1992).

25. F. Y. Yueh and E. J. Beiting, Appl. ()pt. 27, 3233 (1988).

26. J. P. Singh and F. Y. Yueh, Quarterly Technical Progress Report, DIAL Missi sippi State University FE-15601-33 (1989).

27. J. P. Singh and F. Y. Yueh, AIAA Plasmadynamics and Lasers (June 1991).

28. J. P. Singh and F. Y. Yueh, Quarterly Technical Progress Report, DIAL MIssissippi State University FE-15601-40(1990)).

29. J. P. Singh and F. Y. Yueh, R. L. Cook, J. J. Lee and J. T. Lineberry, Appl. Spectrosc. $46,1649(1992)$. 

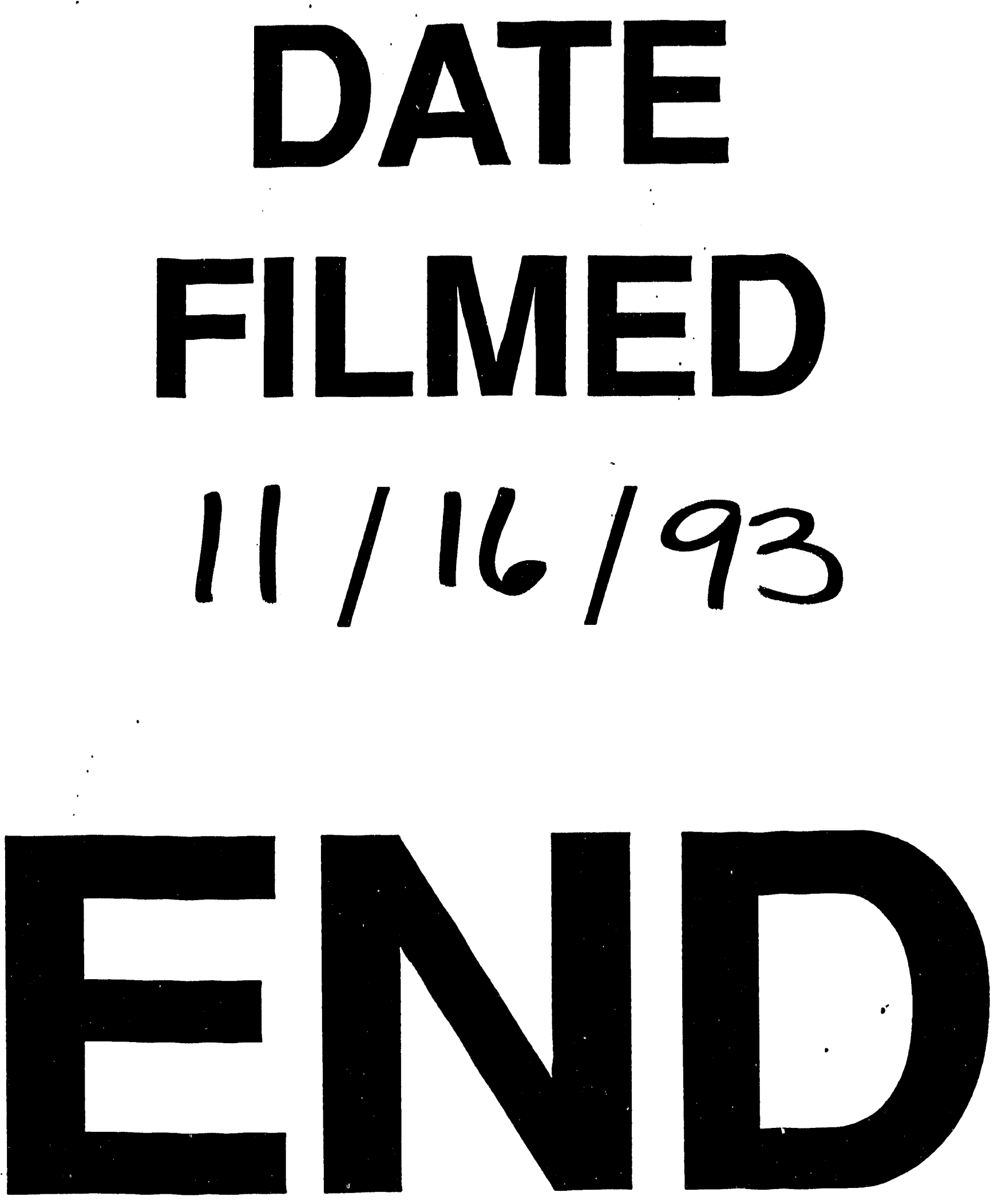
$$
-
$$

$\longrightarrow$
}

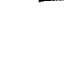
(2) 